\title{
Refractory Bullous Pemphigoid in a Patient with Metastatic Lung Adenocarcinoma Treated with Pembrolizumab
}

\author{
Andrés F. Cardona ${ }^{a, b}$ c Alejandro Ruiz-Patiño ${ }^{a, b}$ \\ Zyanya Lucia Zatarain-Barron $^{d}$ Santiago Ariza ${ }^{\mathrm{e}}$ Luisa Ricaurte ${ }^{f}$ \\ Christian Rolfog ${ }^{9}$ Oscar Arrieta ${ }^{d}$ \\ ancology Department, Foundation for Clinical and Applied Cancer Research (FICMAC), \\ Bogotá, Colombia; ${ }^{b}$ Molecular Oncology and Biology Systems Research Group (FOX-G), \\ Universidad el Bosque, Bogotá, Colombia; 'Clinical and Translational Oncology Group, \\ Clínica del Country, Bogotá, Colombia; ${ }^{\mathrm{d} T h o r a c i c ~ O n c o l o g y ~ U n i t, ~ I n s t i t u t o ~ N a c i o n a l ~ d e ~}$ \\ Cancerología (INCan), Mexico City, Mexico; ${ }^{e}$ Dermatology Oncology Department, Clínica

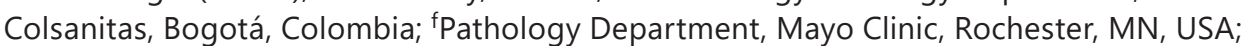 \\ 9Thoracic Oncology Unit, Marlene and Stewart Comprehensive Cancer Center, University of \\ Maryland, Baltimore, MD, USA
}

\author{
Keywords \\ Immunotherapy · Pembrolizumab · Adverse events · Toxicity · Skin reactions
}

\begin{abstract}
We present the case of a 73-year-old male patient with a history of tobacco use who presented with a central nervous system mass that was confirmed to be a lung adenocarcinoma metastasis. High PD-L1 expression as well as negativity to other targetable drivers led to initiation of pembrolizumab monotherapy and ablative stereotactic radiation therapy on oligoresidual disease, achieving a complete response after 2 years of therapy. Following discontinuation of systemic treatment, the patient developed widespread desquamative plaques. A skin biopsy revealed subepidermal blistering and eosinophilic infiltration in conjunction with C3 and IgG depositions on the basement membrane, detected by immunofluorescence. A diagnosis of bullous pemphigoid was obtained, and systemic corticosteroids were administered with lesion progression. Infliximab was also administered without meaningful clinical improvement. Metronomic cyclophosphamide achieved a complete resolution of skin lesions and up to this day the patient continues with tumor control and is free of dermatological findings. In conclusion, bullous pemphigoid is a very rare dermatological adverse effect related with pembrolizumab treatment. Only two cases, including this one, have been reported, especially with this medication for the treatment of non-small cell lung cancer. With more reported cases, management strategies can be optimized even in the steroid refractory setting.
\end{abstract}


Cardona et al.: Refractory Bullous Pemphigoid after Immunotherapy

\section{Introduction}

Immune checkpoint inhibitors (monoclonal antibodies targeting cytotoxic T lymphocyteassociated antigen-4 [CTLA-4], programmed cell death protein 1 [PD-1], or programmed death ligand 1 [PD-L1]) represent a novel class of anticancer agents [1]. Their development represents a major breakthrough in cancer therapy, and they are already registered or are undergoing evaluation in a wide range of advanced cancers. Due to their unique mechanism of action, immune checkpoint inhibitors have a very specific safety profile that is mostly mediated by the triggering of cytotoxic CD4+/CD8+ T-cell activation [2]. More than $60 \%$ of treated patients develop immune-related adverse events (irAEs), which can, theoretically, affect any of the body organs; however, dermatologic toxicities appear to be the most prevalent irAEs.

\section{Case Description}

In February 2017, a 73-year-old male, who was a former heavy smoker, had an episode of herpes zoster in the T12/11 dermatomes followed by dizziness and disorientation. A brain MRI described an expansive intra-axial lesion of peripheral location at the left parasagittal parieto-occipital junction with compromise of the precuneus and cuneal gyrus. This finding presented oval morphology with irregular annular peripheral enhancement, measuring $29 \times$ $24 \times 28 \mathrm{~mm}$ and was associated with surrounding edema. He was taken to surgery finding a poorly differentiated malignant tumor with reactivity for $\mathrm{Ck}_{7}$, Napsin $\mathrm{A}$, and $\mathrm{PDL}_{1}(>50 \%)$ compatible with a lung adenocarcinoma. Extensive genomic evaluation by NGS identified no alterations in $E G F R, A L K$, and ROS; however, the panel revealed the presence of the mutation $K R A S^{G 12 D}$. Subsequently, a postoperative brain MRI found no residual disease and a PET-CT showed an abnormal increase in the FDG uptake (SUVmax 10.5) in a mass located in the posterior segment of the lower lobe (43 $\times 25 \times 32 \mathrm{~mm} ; \mathrm{T}_{2 \mathrm{~b}} \mathrm{~N}_{0} \mathrm{M}_{1 \mathrm{~b}} \mathrm{G}_{3}$ stage IV). In this same lung, another small poorly defined nodular image and a well-defined rounded nodule in the right lower lobe of $8 \mathrm{~mm}$ were observed. Twenty days after the neurosurgical intervention, radiosurgery was performed on the cavity followed by pembrolizumab (200 mg every 21 days), a management with which he achieved partial response after 20 cycles. In June 2018, SBRT was performed on oligoresidual lung disease $(18 \times 21 \mathrm{~mm}$ node $)$, continuing the antiPD-1 until 35 cycles ( 2 years), which ended in complete response during July 2019. In terms of irAEs, the patient presented a grade 2 pneumonitis and a relative adrenal insufficiency that required the transient use of steroids, the latter upon completing 25 cycles of pembrolizumab.

In November 2019, 4 months after finishing immunotherapy, the patient developed a moderate-to-severe itching reaction around the thorax, arms, and hips. On examination, he had ill-defined desquamative erythematous plaques over the described areas. An initial punch skin biopsy was performed on the left arm, revealing an epidermis with mild spongiosis, irregular acanthosis, and focal exocytosis of lymphocytes and eosinophils, whereas in the dermis there was mild superficial perivascular chronic inflammation with eosinophilic infiltration. The patient was treated with twice daily topical betamethasone, oral antihistamine treatment, and a 14-day course of oral prednisone $(1 \mathrm{mg} / \mathrm{kg} \mathrm{q} /$ day), experiencing a transient improvement in skin manifestations. A few weeks later, during patient reassessment he developed persistent eczematous plaques extending over the back, chest, thighs, and lower legs, associated with severe pruritus, which significantly affected his quality of life. Three weeks later, the patient was admitted to the hospital due to extensive erythematous papules and plaques, in addition to a few intact and ruptured vesicles and bullae over the upper and lower limbs, especially over the knees, elbows, palms, and soles of hands and feet (Fig. 1).

\section{Karger'}



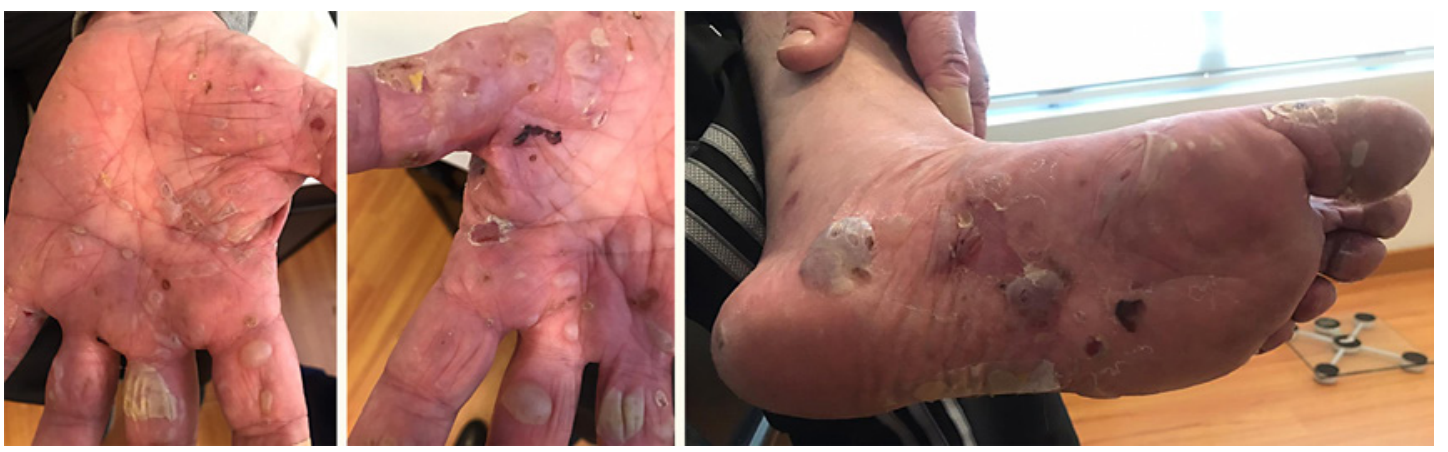

Fig. 1. Tense bullae arise on skin surface, with a predilection on the flexural areas of the upper and lower limbs.
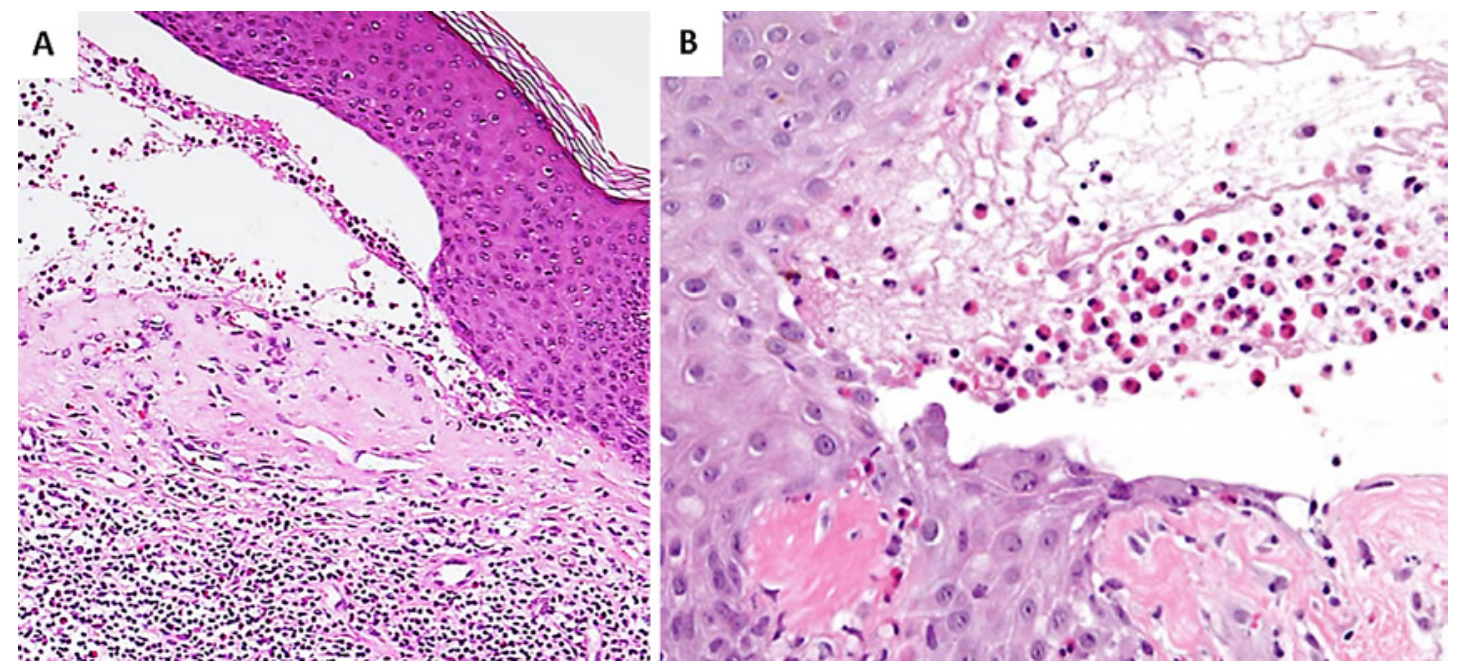

Fig. 2. A Subepidermal blister. The inflammatory infiltrate is polymorphous, with an eosinophil predominance. Linear deposition of C3 and IgG along the basement membrane zone. Hematoxylin and eosin. B Superficial inflammation with numerous eosinophils. Early cleft formation.

A new skin biopsy was subsequently taken from the left foot, for hematoxylin and eosin staining and direct immunofluorescence studies. Pathology analysis confirmed subepidermal blisters with eosinophilic infiltration. The overlying epidermis showed early degenerative changes and parakeratosis. There was moderate inflammation in the papillary dermis, consistent with the presence of multiple lymphocytes and eosinophils (Fig. 2A, B). Immunofluorescence microscopy showed noncontinuous linear deposition of IgG at the dermoepidermal junction (not available) and continuous linear deposition of $\mathrm{C} 3$ at the dermoepidermal junction. The diagnosis was thus consistent with bullous pemphigoid (BP). Accordingly, the patient was treated with intravenous methylprednisolone $2 \mathrm{mg} / \mathrm{kg} /$ day without improvement (after 5 days) and with progressive involvement of the oral mucosa and throat, a finding that was associated with limitation for intake and mild hyperthermia.

Due to refractory behavior of the pembrolizumab-induced BP to corticosteroid treatment, we administered $5 \mathrm{mg} / \mathrm{kg}$ infliximab with transitory pain improvement without any changes in ulcer distribution. Based on retrospective reports and the current ESMO guideline for toxicity induced by anti-CTLA-4/PD-1/L1 antibodies, a second dose was placed 2 weeks after the first administration without finding greater improvement or a clear difference in the previously described symptoms. Based on this, oral metronomic cyclophosphamide was 
initiated using $50 \mathrm{mg}$ every day by 21 days in a regimen of 28 days for 2 cycles. After the first month, we found a rapid improvement in the cutaneous findings, disappearance of the pain, and almost complete regression of the pruritus. Currently, the patient is without active cancer treatment, maintains the complete response, and satisfactorily modulates the BP.

\section{Discussion}

$\mathrm{BP}$ has been anecdotally reported in the literature as a rare manifestation of immunological-mediated toxicity. Due to shifting an immunological imbalance favoring autoimmunity, acute flares of previously controlled autoimmune disorders have been previously reported. Such was the case for one patient treated with pembrolizumab for metastatic urothelial carcinoma, who developed BP after the initial cycles. Although treatment with the anti-PD-1 antibody was suspended, the skin lesions persisted and were refractory to some extent to systemic and topical corticosteroids [3]. Other cases presented in the literature of patients treated with pembrolizumab are strongly associated with other neoplasms, especially melanoma. Association with non-small cell lung cancer has only been documented, to the best of the authors' knowledge, in one case, who presented with severe and extensive involvement resulting in widespread erosions [4].

Histological findings of C3 and IgG depositions along the basement membrane determined by immunofluorescence are present both in pembrolizumab-induced and primary BP, indicating a similar pathophysiological mechanism. Furthermore, identification of both anti-BP230 and anti-BP180 antibodies in both conditions confirms that these entities are both clinically and pathologically undistinguishable [4]. The specific alteration that leads to the development of this form of toxicity is still unknown. Hypotheses can be drawn from a cohort study conducted in the Mayo Clinic in which different asymptomatic individuals were evaluated for the presence of the aforementioned antibodies resulting in a $7.4 \%$ positivity rate. These results also suggest that the presence of these antibodies is not predictive of BP episodes, but rather that external factors are requisite, which modulate its development [5]. Taking into consideration that the PD-1 pathway plays an important role in co-stimulatory inhibition of T-cell homeostasis, both as a peripheral and central tolerance regulator, its loss of function has been linked to the development of several autoantibodies and acceleration of autoimmune features [6].

Time of exposure and development of BP also varies. Both early onset after initiation and late onset after discontinuation have been reported [7]. Taking into account the previous considerations, we suggest that early-onset BP is associated with already present antibodies that manifest after peripheral tolerance is inhibited, whereas late-presenting BP could be associated with the loss of central tolerance and the subsequent development of autoantibodies.

Management of such a rare adverse effect is also a clinical challenge. Several cases have been managed with both systemic and topical steroids with success of lesion resolution obtained in some degree, with the majority of cases achieving a satisfying response to initial therapy [7]. In the presented case, refractoriness to steroid therapy was documented and infliximab initiated. Since no response to treatment was observed, administration of cyclophosphamide was required. Although it is not recommended as a first-line treatment, even for primary BP, this medication offers a clinically significant benefit with good tolerance, especially in elderly individuals. Since no clear recommendations exists beyond steroids, it should be considered as an alternative treatment.

Administration of immunosuppressant medications, especially steroids, is associated with worse prognosis with regards to tumoral control and should be taken into consideration when opting for their administration, especially when accompanied by suspension of immune checkpoint inhibitors [8].

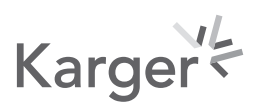




\section{Conclusion}

In conclusion, pembrolizumab associated BP in lung cancer is a very rare adverse event, which has been documented in only two occasions, including the presented case. Pathophysiology as well as management are similar to primary or idiopathic BP, with steroid refractoriness being a relevant and considerable clinical course, requiring immunomodulatory medications. Widespread knowledge, especially in recognizing and treating these rare manifestations, is important for their adequate management.

\section{Statement of Ethics}

Written informed consent was obtained for publication of this case report and any accompanying images by the patient's next of kin.

\section{Conflict of Interest Statement}

The authors have no conflicts of interest to declare.

\section{Funding Sources}

There was no funding received for this study.

\section{Author Contributions}

Conceptualization: AFC. Data review: AFC. Dermatological assessment: SA; LR. Oncological follow-up: AFC, CR, OA. Writing original draft: AFC, ARP, ZLZB, SA, LR, CR, OA. Writing review and editing: AFP, ARP, ZLZB. Final approval: AFC, ARP, ZLZB, SA, LR, CR, OA.

\section{References}

1 Postow MA, Callahan MK, Wolchok JD. Immune Checkpoint Blockade in Cancer Therapy. J Clin Oncol. 2015; 33(17):1974-82.

2 Naidoo J, Page DB, Li BT, Connell LC, Schindler K, Lacouture ME, et al. Toxicities of the anti-PD-1 and anti-PDL1 immune checkpoint antibodies. Ann Oncol. 2015;26(12):2375-91.

3 Garje R, Chau JJ, Chung J, Wanat K, Zakharia Y. Acute Flare of Bullous Pemphigus With Pembrolizumab Used for Treatment of Metastatic Urothelial Cancer. J Immunother Hagerstown Md. 1997;41(2018):42-4.

4 Qiu C, Shevchenko A, Hsu S. Bullous pemphigoid secondary to pembrolizumab mimicking toxic epidermal necrolysis. JAAD Case Rep. 2020;6(5):400-2.

5 Wieland CN, Comfere NI, Gibson LE, Weaver AL, Krause PK, Murray JA. Anti-bullous pemphigoid 180 and 230 antibodies in a sample of unaffected subjects. Arch Dermatol. 2010;146(1):21-5.

6 Francisco LM, Sage PT, Sharpe AH. The PD-1 pathway in tolerance and autoimmunity. Immunol Rev. 2010; 236:219-42.

7 Lopez AT, Khanna T, Antonov N, Audrey-Bayan C, Geskin L. A review of bullous pemphigoid associated with PD-1 and PD-L1 inhibitors. Int J Dermatol. 2018;57(6):664-9.

8 Pan EY, Merl MY, Lin K. The impact of corticosteroid use during anti-PD1 treatment. J Oncol Pharm Pract. 2020; 26(4):814-22. 